\title{
Patterns of Growth and Body Proportions After Total-Body Irradiation and Hematopoietic Stem Cell Transplantation During Childhood
}

\author{
BOUDEWIJN BAKKER, WILMA OOSTDIJK, RONALD B. GESKUS, W. HENRIËTTE STOKVIS-BRANTSMA,
} JAAK M. VOSSEN, AND JAN M. WIT

\author{
Department of Pediatrics [B.B., W.O., W.H.S.-B., J.M.V., J.M.W.], Department of Medical Statistics [R.B.G.], Leiden University Medical \\ Center, Albinusdreef 2, 2333 ZA, Leiden, The Netherlands
}

\begin{abstract}
Patterns of growth and body proportions were studied in 75 children receiving total-body irradiation (TBI) and hematopoietic stem cell transplantation (SCT) before onset of puberty. Of the 19 patients receiving $\mathrm{GH}$, only data obtained before onset of $\mathrm{GH}$ were included. Thirty-two patients reached final height (FH). Median change in height SD score (SDS) between SCT and FH was -1.7 in boys and -1.1 in girls. Peak height velocity (PHV) was decreased in the majority of the patients (median PHV $5.7 \mathrm{~cm} / \mathrm{y}$ in boys and 5.3 $\mathrm{cm} / \mathrm{y}$ in girls), even though it occurred at appropriate ages. Changes in body proportions were analyzed by linear mixed-effects models. Decrease in sitting height SDS did not differ between boys and girls. In boys, decrease in leg length SDS was of comparable magnitude, whereas, in girls, decrease in leg length was less pronounced, leading to a significant decrease in SDS for sitting height/height ratio in girls only. The sex-specific effects of several variables on height SDS were analyzed by linear mixed-effects modeling, showing a slightly faster decrease in younger children and a more pronounced decrease during puberty in boys compared with girls. We conclude that 1) younger children are more susceptible to growth retardation after TBI and SCT, 2) pubertal growth is more compromised in boys, and 3) leg growth is relatively less affected in girls, possibly due to a high incidence of gonadal failure in girls. (Pediatr Res 59: 259-264, 2006)
\end{abstract}

$\mathrm{H}$ ematopoietic SCT has become a standard treatment option for many children with congenital or malignant disorders of the hematological system. The intensive conditioning regimens required in most cases often result in impaired growth and reduced final height, especially if unfractionated TBI is part of the conditioning regimen (1). TBI can impair growth both directly, by damaging the epiphyseal growth plates, and indirectly, by decreasing GH secretion, or by causing hypogonadism or hypothyroidism (2). Other factors that may contribute to growth delay are chronic graftversus-host disease in recipients of allogeneic transplants, use of corticosteroids, psychosocial dysfunction, and insufficient nutritional intake.

Received May 17, 2005; accepted September 21, 2005

Correspondence: Boudewijn Bakker, M.D., Department of Paediatrics, Leiden University Medical Center, PO Box 9600, 2300 RC Leiden, The Netherlands: e-mail: b.bakker@lumc.nl

This work was sponsored by a grant from NWO, The Netherlands' Organization for Scientific Research (grant number 920-03-034).

DOI: 10.1203/01.pdr.0000199550.71887.ba
In contrast to the extensively documented negative effect of TBI and SCT on height, more recently completed with data on final height (1,3-8), the effect of TBI and SCT on other aspects of growth are less intensively studied. To our knowledge, for example, height development after TBI and SCT (including influences of sex and puberty) has not yet been modeled, and the effect on body proportions has been the subject of only a few studies (5,9-12). Moreover, most of these studies included patients who had received cranial irradiation before TBI, patients who had already entered puberty at the time of SCT, or patients who had been treated with GH. In addition, none of these studies considered sex differences.

In an attempt to clarify these aspects of growth after SCT, we investigated sex-specific development of height, body proportions, and final height in children receiving TBI and SCT for hematological malignancies before the onset of puberty. Patients who had received cranial irradiation were excluded, as well as height measurements taken after onset of $\mathrm{GH}$ therapy. In addition, we constructed sex-specific models of growth after TBI and SCT.

\section{PATIENTS AND METHODS}

Between 1980 and 2001, 193 prepubertal children with a hematological malignancy received TBI-based conditioning for SCT at the Leiden University Medical Center. All children without a relapse after SCT, who had more than $2 \mathrm{y}$ of follow-up after TBI-based conditioning for SCT for a hematological malignancy before the onset of puberty were selected for this study $(n=$ $89)$. We excluded patients who had received cranial irradiation $(n=6)$ or had developed chronic graft-versus-host disease $(n=7)$ or had Down syndrome $(n=1)$. A total of 75 patients were included; their characteristics are summarized in Table 1. Puberty onset had occurred in 61 patients (40 males). Serum level of IGF-I and IGF binding protein 3 were measured annually in all patients, and patients were tested for GH deficiency if growth was impaired. GH therapy was given to 19 patients (13 males). Indications for GH therapy were GH deficiency $(n=1)$, GH neurosecretory dysfunction $(n=2)$, and growth impairment $(n=16)$. Only data obtained before start of GH therapy were included.

The study was approved by the by the Institutional Review Board (Leiden University Scientific Review Board) and the Review Board of the Netherlands' Organization for Scientific Research (NWO).

Conditioning for SCT. Conditioning for SCT consisted of TBI and cyclophosphamide in all patients. In addition to cyclophosphamide $(60 \mathrm{mg} /$ $\mathrm{kg} / \mathrm{d}$ i.v. for 2 consecutive days), cytarabine $\left(1 \mathrm{~g} / \mathrm{m}^{2} / \mathrm{d}\right.$ for 2 consecutive

Abbreviations: FH, final height; PHV, peak height velocity; SCT, stem cell transplantation; SDS, standard deviation score; TBI, total-body irradiation 
Table 1. Population characteristics

\begin{tabular}{lcc}
\hline & Male & Female \\
\hline No. of patients & 53 & 22 \\
Age at SCT & $8.2(2.3-13.9)$ & $7.2(0.8-12.3)$ \\
Age puberty (40 male/21 female) & $12.3(9.8-14.5)$ & $10.9(9.0-14.1)$ \\
Follow-up (in years) & $8.4(2.0-15.5)$ & $11.0(3.5-20.2)$ \\
Gonadal failure before age 15 y & 8 & 11 \\
SCT Type & & \\
Autologous & 4 & 2 \\
Identical related & 37 & 16 \\
Matched unrelated & 8 & 3 \\
Haploidentical & 4 & 1 \\
Diagnosis & & \\
Acute lymphocytic leukemia & 26 & 7 \\
Acute myelogenous leukemia & 18 & 12 \\
MDS & 5 & 2 \\
Non-Hodgkin lymphoma & 2 & 1 \\
Chronic myelogenous leukemia $(\mathrm{Ph}+)$ & 2 & - \\
TBI dose & & \\
5.0 Gy & - & 2 \\
7.0 Gy & 8 & 3 \\
7.5 Gy & 26 & 12 \\
8.0 Gy & 7 & 2 \\
Testicular booster $4 \times 2.5$ Gy & 12 & 3 \\
\hline
\end{tabular}

MDS, myelodysplastic; n.a., not applicable.

days) was given to patients treated for myeloid leukemia or MDS between 1988 and $1998(n=20)$. From 1990 onward, patients treated for lymphoblastic leukemia or non-Hodgkin lymphoma $(n=32)$ received etoposide ( $350 \mathrm{mg} / \mathrm{m}^{2} / \mathrm{d}$ for 2 consecutive days) in addition to cyclophosphamide. All patients received unfractionated TBI, delivered at a mean dose rate of $25 \mathrm{cGy} / \mathrm{min}$. As age is an important determinant of the tolerable total irradiation dose in children, a TBI regimen with age-dependent total dose was applied (0-2 y: $5.0 \mathrm{~Gy} ; 2-4$ y: $7.0 \mathrm{~Gy} ; 4-10$ y: $7.5 \mathrm{~Gy} ;>10$ y: $8.0 \mathrm{~Gy})$. The latter dose was "increased" in 1989 to two single fractions of $6.0 \mathrm{~Gy}$, given on 2 consecutive days (instead of the equivalent 9.0 Gy once, which had too many side effect in adults). Eight boys received additional prophylactic testicular irradiation (10 Gy in four fractions before TBI).

Auxological parameters. Data on height, sitting height, Tanner stages of breast or genital development (13), and testicular volume (measured with an orchidometer) were collected from the clinical records. Subischial leg length was defined as height minus sitting height. The ratio between sitting height and height was used as parameter for body proportions. Auxological parameters were expressed as SDS for sex and age, all based on the same reference population $(n=14,500)$ from the 1997 Dutch Growth Study $(14,15)$. Patients were considered to have reached final height if height velocity was less than $1 \mathrm{~cm} / \mathrm{y}$ in pubertal subjects.

Puberty and gonadal function. The onset of puberty was defined as the age at which breast development was first recorded in girls (Tanner breast stage $\geq B 2$ ) or a testicular volume $\geq 4 \mathrm{~mL}$ was reached in boys. If signs of pubertal development (e.g. penile growth and pubic hair development) had occurred in boys whereas testicular volume had not reached $4 \mathrm{~mL}$, onset of puberty was determined on the basis of the combination of progression of Tanner stages and increasing serum levels of testosterone. If puberty was induced, onset of puberty was defined as the onset of sex hormone replacement therapy. In girls, hypergonadotropic hypogonadism was used as a parameter of ovarian failure. In boys, elevated serum levels of LH with or without decreased serum levels of testosterone were used as a parameter for Leydig cell failure, and elevated levels of FSH were used as an indicator of Sertoli cell failure (as a result of germ cell loss and/or Sertoli cell loss).

Statistical analyses. S-PLUS 6 Professional (Insightful Corp., Seattle, WA) was used for all statistical analyses, with significance level set at 0.05 .

A height velocity curve was fitted by monotone smoothing procedure as described by Ramsay et al. $(16,17)$ for each individual who had at least five height measurements between 8 and 16 y of age for girls $(n=16)$ or 10 and 18 y of age for boys $(n=29)$, and PHV and age at PHV were calculated. We used penalized least squares with a penalty that depended on the number of measurements (penalty $250 / N^{2}$, with $N=$ the number of individual measurements).
Changes in SDS for the different auxological measurements with time since transplantation were fitted by mixed-effects models with data grouped by individual patients, SDS as dependent variable, and time since SCT as major independent variable (see next paragraph for background information on mixed-effects models). We used the function "Ime" (linear mixed-effects) in S-PLUS.

Choice of analyzing methods. The analysis of growth after SCT is a typical example of a repeated measurements analysis, in which both the number of observations and the interval between the observations may vary between the patients (i.e. both time between visits and duration of follow-up may vary). A traditional standard linear regression analysis assumes all measurements to be independent and may yield biased results, especially if the data are unbalanced. An alternative is to fit a growth curve separately for each individual and average the patterns. This has been done for the height velocity analyses but has the disadvantage that estimated patterns are very uncertain for individuals with few measurements. Therefore, we only used data of patients with at least five measurements in the pubertal age for the construction of the pubertal height velocity curves.

Mixed-effects models also fit a growth curve for each individual, after which the parameters for the growth curves are averaged to obtain a population effect. However, for persons with relatively little information, information is used from the other individuals to obtain their growth curve parameters. This is obtained by assuming that the growth curve parameters are a random sample from some distribution (usually a normal distribution), and the parameters are included as "random effects." Moreover, the effects of some covariates can be chosen to be equal for all individuals, and these covariates are only included as "fixed effects." The use of mixed-effects models (combining random and fixed effects) allows the inclusion of all available data in the analyses without the risk of considerable bias. As far as we know, mixed-effects models have not been used before in the analysis of growth after SCT.

Identification of factors influencing height SDS after SCT in the mixedeffects model. We used time, sex, puberty, age at SCT, and gonadal failure as independent variables and height SDS after SCT as dependent variable for our model. The basis of our model was a linear change (decrease) in height SDS with time since SCT. As patients were recovering from their illness, we allowed the slope to be different in the first $2 \mathrm{y}$ after SCT compared with the following years. The value of the intercept and the slopes in the first $2 \mathrm{y}$ after SCT and in the years thereafter differed per individual, as was expressed by their random effects. The slope of the major time variable was allowed to depend on age at SCT, sex, and the presence of gonadal failure before the age of $15 \mathrm{y}$. The effect of gonadal failure was allowed to depend on sex.

The effect of puberty on height SDS was divided into two components. The first component is a reference population effect: when patients from the same sex reach the pubertal age (approximately $8.5 \mathrm{y}$ in girls and $10.5 \mathrm{y}$ in boys) height velocity of the reference population gradually increases as a result of the pubertal growth spurt and subsequently decreases. If the child itself has not yet reached puberty, this will cause its height SDS to decrease in a nonlinear way. The second component of the effect of puberty is the individual pubertal growth spurt of our patients, which will result in a gradual increase in height SDS after onset of puberty, which stabilizes after 3-5 y. In patients with an early onset of puberty, height SDS may even decrease at the end of their growth spurt. As this second component of puberty is an individual effect, which may vary between patients, it was included as a random effect as well. We allowed both puberty effects to depend on sex. We used sigmoid curves (logit transformations) to model both puberty effects. Formula 1 represents the relation for the sigmoid curve of the population effect, with $x_{1}$ being the time in years since reaching the pubertal age ( 8.5 in girls and 10.5 in boys). Formula 2 represents the relation for the sigmoid curve of the individual puberty effect, with $x_{2}$ being the time since individual onset of puberty in years. The second part of both formulas is a correction forcing the results to equal zero at $\mathrm{x}=0$.

$$
\text { Formula 1: } \beta 1\left(\frac{e^{\left(x_{1} \cdot \text { scale }-2\right) \cdot 2}}{1+e^{\left(x_{1} \cdot \text { scale }-2 \cdot 2\right)}}\right)-0.01798621
$$

Formula 2: $\beta 2\left(\frac{e^{\left(x_{2} \cdot \text { scale }\right)}}{1+e^{\left(x_{2} \cdot \text { scale }\right)}}\right)-0.5$

The optimal scale parameter, which is included in the model in a nonlinear way, was obtained by fitting the model for an array of values of "scale." The combination with the best Bayesian information criterion (BIC) value was chosen. 


\section{RESULTS}

Height and height velocity. A total of 974 height measurements from 75 individuals contributed to the results. Figure 1 represents the smoothened individual curves of height velocity of 29 males and 14 females. In four patients (three girls), no pubertal growth spurt could be detected; all showed an attenuation of decrease in height velocity between 10.0 and $12.5 \mathrm{y}$ of age. Of the patients with a detectable pubertal growth spurt, median pubertal PHV was $5.7 \mathrm{~cm} / \mathrm{y}$ in boys $(n=29$; range, $3.5-8.6 \mathrm{~cm} / \mathrm{y})$ and $5.3 \mathrm{~cm} / \mathrm{y}$ in girls $(n=14$; range, $3.1-$ $7.3 \mathrm{~cm} / \mathrm{y})$. Median age at PHV was $13.4 \mathrm{y}(10.1-15.4)$ in boys and 10.9 y $(8.6-14.0)$ in girls.

FH was reached by 32 ( 21 males) of the 56 patients who had not received GH. Of the patients who had reached FH, age and height SDS at time of SCT, at onset of puberty and at final height are summarized in Table 2.

Body proportions. Since 1991, sitting height was measured on a routine basis as part of the follow-up protocol. A total of 373 sitting height measurements from 69 patients (19 females) contributed to the results. The results of the linear mixedeffects models for changes in SDS with time since SCT for the different auxological measurements are summarized in Table 3. In males, there was a significant decrease in both sitting height SDS and leg length SDS, and these changes were of comparable magnitude. Therefore, the SDS for the sitting height/height ratio did not change. In females, however, the significant reduction in sitting height SDS was not accompanied by a significant reduction in leg length SDS, resulting in a significant decrease in sitting height/height ratio SDS. The decrease in sitting height/height ratio SDS was more pronounced in the 10 girls with ovarian failure before the age of 15 y (estimate $-0.117 \mathrm{SD} / \mathrm{y} ; p<0.0001 ; \mathrm{SE}=$ $0.017)$ compared with the 9 girls without ovarian failure $(-0.032 \mathrm{SD} / \mathrm{y} ; p=0.49 ; \mathrm{SE}=0.046)$. In boys, no differences were detected between the 8 boys with Leydig cell failure (all had received additional testicular irradiation) and the 42 boys without Leydig cell failure.

Model for changes in height SDS after SCT. The final model is summarized in Table 4 and the resulting curves are represented in Figure 2. The model describes a linear decrease

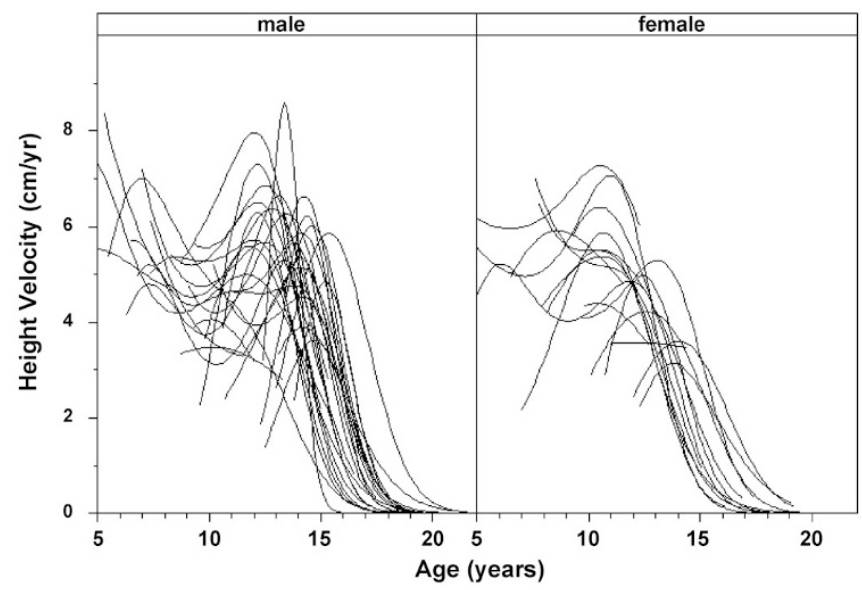

Figure 1. Smoothed individual curves for height velocity of males $(n=29)$ and females $(n=14)$.
Table 2. Age and height SDS at different times between SCT and FH of the 32 patients who have reached final height

\begin{tabular}{lcc}
\hline & Male $(n=21)$ & Female $(n=11)$ \\
\hline Age (y) & & \\
SCT $\dagger$ & $10.9(5.6-13.9)$ & $8.2(0.8-12.3)$ \\
Puberty onset* & $13.2(10.0-14.5)$ & $11.2(10.0-14.1)$ \\
Final height* & $18.0(15.4-20.2)$ & $17.4(16.0-19.2)$ \\
Time (y) & $1.5(0.3-5.6)$ & $2.8(1.2-9.7)$ \\
SCT to puberty onset & $5.6(2.6-7.3)$ & $5.1(3.9-7.8)$ \\
Puberty onset to final height & & \\
Height (SDS) & $+0.4(-1.7$ to +1.3$)$ & $-0.2(-0.5$ to +0.9$)$ \\
Target height & $-0.6(-1.9$ to +0.9$)$ & $-0.6(-1.9$ to +1.5$)$ \\
SCT & $-0.8(-2.3$ to +0.9$)$ & $-1.4(-2.4$ to +1.1$)$ \\
Puberty onset & $-1.8(-3.7$ to +0.1$)$ & $-1.6(-3.1$ to +0.5$)$ \\
Final height & & \\
Height (cm) & $186.9(171.7-193.0)$ & $169.5(167.1-176.3)$ \\
Target height $\$$ & $171.0(157.6-184.4)$ & $159.9(150.7-173.8)$ \\
Final height** & & \\
Height differences (SDS) & $-0.1(-1.1$ to +0.7$)$ & $-0.5(-1.2$ to +0.0$)$ \\
Puberty onset—SCT* & $-1.7(-3.4$ to -0.0$)$ & $-1.1(-2.2$ to -0.0$)$ \\
Final height—SCT & & \\
Final height—target height & $-2.1(-4.5$ to -0.0$)$ & $-1.4(-2.8$ to +0.1$)$ \\
Height differences (cm) & & \\
Final height—target height & $-14.9(-32.1$ to -0.2$)$ & $-9.3(-18.2$ to +0.4$)$ \\
\hline
\end{tabular}

Data represented as median (range). Patients treated with GH are excluded from analyses.

Significant differences between boys and girls (Wilcoxon rank sum test) are marked by symbols: $\dagger p=0.051 ; * p<0.05$; ** $p<0.01 ; \S p<0.001$.

Table 3. Results of linear mixed-effects models for changes in SDS with time since SCT

\begin{tabular}{llll}
\hline & & \multicolumn{2}{c}{ Change in SDS per year since SCT } \\
\cline { 3 - 4 } & & $\begin{array}{c}\text { Males } \\
(\mathrm{n}=50)\end{array}$ & $\begin{array}{c}\text { Females } \\
(\mathrm{n}=19)\end{array}$ \\
\hline Height & $-0.482^{*}$ & $-0.136^{* *}$ & $-0.112^{* *}$ \\
Sitting height & $-0.145 \S$ & $-0.147^{* *}$ & $-0.149^{* *}$ \\
Leg length & $-0.609^{* *}$ & $-0.117^{* *}$ & $-0.021 \S$ \\
Sitting height:height & $-0.463^{* *}$ & $+0.002 \S$ & $-0.094^{* *}$ \\
\hline
\end{tabular}

If The intercepts did not differ between males and females.

$\S$ Not significant.

$* p<0.001$.

$* * p<0.0001$.

in height SDS with time after SCT. This decrease did not differ significantly ( $p=0.32$ ) between boys and girls (Fig. 2, $a$ and $d$ ). For boys receiving SCT at the age of 4 , the decrease per year was $-0.13 \mathrm{SD} / \mathrm{y}(p<0.0001)$, whereas for girls it was $-0.16 \mathrm{SD} / \mathrm{y}$. For each year increase in age at SCT, this decrease in height SDS is $0.009 \mathrm{SD} / \mathrm{y}$ less $(p=0.01)$. The effect of reaching the population pubertal age (i.e. $8.5 \mathrm{y}$ in girls and $10.5 \mathrm{y}$ in boys) was best described by a combination of a linear increase in height SDS $(0.062 \mathrm{SD} / \mathrm{y}$ for males, 0.076 for females) and a logistic decrease in height SDS with $\beta 1=-1.9$ for males and -1.2 for females, and scale parameter 0.4 (see formula 1 and Fig. 2, $b$ and $e$ ). The decrease in height SDS caused by reaching the pubertal age was significantly greater in boys compared with girls $(p=0.0007)$.

A modest pubertal growth spurt could be detected in both boys and girls, with a logistic increase in height SDS after onset of puberty with $\beta 2=0.72$ for males and 1.17 for 
Table 4. Summary of the model of change in height SDS after TBI $(n=75)$

\begin{tabular}{|c|c|c|c|}
\hline & Value & 95\% Confidence interval & $p$ Value \\
\hline Height SD at transplant & -0.49 & -0.72 to -0.26 & $<0.0001$ \\
\hline Time since SCT male & -0.13 & -0.17 to -0.084 & $<0.0001$ \\
\hline Idem female to male* & -0.031 & -0.093 to 0.03 & 0.3211 \\
\hline Age at SCT (from 4 y onward) & 0.009 & 0.0022 to 0.016 & 0.0100 \\
\hline Change slope at onset of puberty in reference population male & 0.062 & 0.0089 to 0.11 & 0.0227 \\
\hline Idem female to male* & 0.014 & -0.056 to 0.085 & 0.6887 \\
\hline Time since onset puberty individual patient ( $\beta 2$ in formula 2 ) male & 0.72 & 0.23 to 1.2 & 0.0047 \\
\hline Idem female to male* & 0.45 & -0.34 to 1.2 & 0.2702 \\
\hline
\end{tabular}

All time parameters are expressed in years.

* Difference between females and males.

$\S$ See Figure 2 for visualization of effects.

Overall $p$ values for effects of time since transplant, onset of puberty in the reference population, onset of individual puberty, and sex were all $<0.0001$. The overall effect of sex on population start puberty had $p=0.0007$.
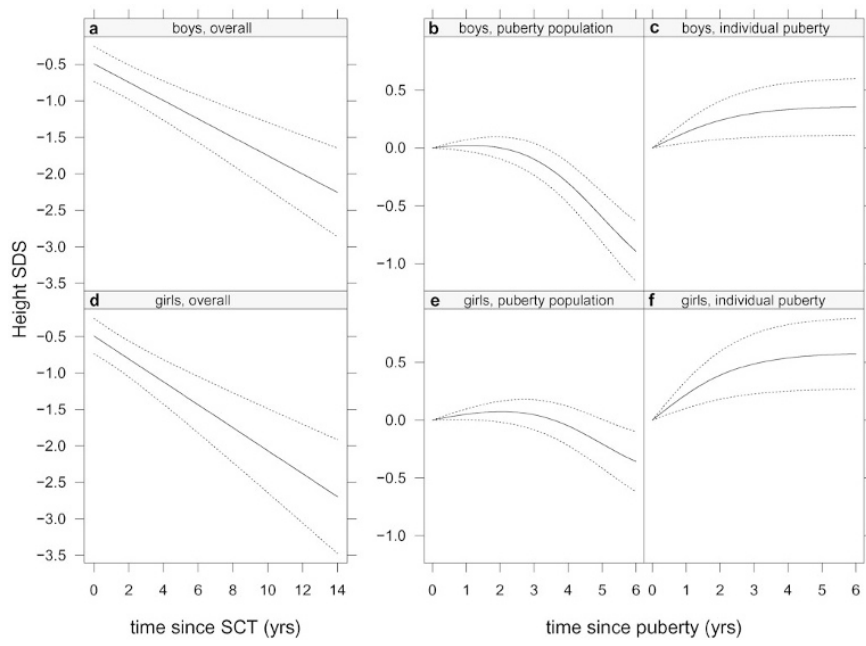

Figure 2. Graphic representation of several components of the model for growth after TBI-based conditioning for SCT, based on all 75 patients (dotted lines represent the $95 \%$ confidence intervals). The upper three panels $(a, b, c)$ represent the males, the lower three panels $(d, e, f)$ females. The panels on the left $(a, d)$ represent change in height SDS with time since SCT. The panels in the middle $(b, e)$ represent change in height SDS with time since onset of puberty in the reference population (10.5 y in boys and $8.5 \mathrm{y}$ in girls). The panels on the right $(c, f)$ represent changes in height SDS with time since the individual onset of puberty.

females, and scale parameter 0.8 (see formula 2, Fig. 2, $c$ and $f$ ). The difference in growth spurt between boys and girls was not significant $(p=0.27)$.

Total TBI dose was determined by age at SCT. The effect of age at SCT was opposite to and dominant over the effect of total TBI dose. Therefore, total TBI dose was not included in the final model. "Gonadal failure" was not included in the final model, as it did not significantly improve the fit.

\section{DISCUSSION}

The present study is one of the largest single-center study to date on growth and final height after TBI-based conditioning for SCT. In addition, it is the first study to analyze the effects of unfractionated TBI on body proportions in boys and girls separately, thereby identifying sex differences in development of body proportions after single-dose TBI. By using the mixed-effects model approach, we were able to identify several factors that influence growth after TBI-based conditioning for SCT.

Pubertal growth spurt. Reference data on height velocity in Dutch children are not available. Beunen and Malina (18) summarized the results of 20 studies on pubertal PHV, in which mean PHV ranged from 8.2 to $10.3 \mathrm{~cm} / \mathrm{y}$ for males and from 7.0 to $9.1 \mathrm{~cm} / \mathrm{y}$ for females. The mean age at PHV in these studies ranged from 13.4 to 14.4 in males and from 10.2 to 12.6 in females. Although our patients reached PHV at appropriate ages, PHV was lower in the vast majority of them. We therefore conclude that the pubertal growth spurt is blunted in our patients. In the patients who had reached final height, the age at PHV was only slightly higher than the age at onset of puberty. As patients visited the clinic only once or twice a year, there might have been a delay of up to $12 \mathrm{mo}$ in the appreciation of onset of puberty by clinical definition. In addition, testicular volume as indicator of onset of puberty may be less suitable in our patients, as testicular volume is compromised after TBI (6).

Final height. Of the 75 patients included in the present study, 32 had reached final height without receiving GH therapy. The decrease in height SDS between TBI and final height in these patients was 1.1 SD in girls and 1.7 SD in boys, which is comparable to that described in most studies on final height after SCT (i.e. $1.0-1.5 \mathrm{SD})(1,3,4,7,8)$. Sf-TBI is generally believed to have a much greater impact on growth than does fractionated TBI (f-TBI) (19). This belief is largely based on studies in patients receiving higher doses (9-10 Gy) sf-TBI, and these studies did not include final height. Recently, several single-center studies reported final height data after TBI in prepubertal patients who had not received additional cranial irradiation. Frisk et al. (7) used 7.5 Gy sf-TBI and reported a median decrease in height SDS of 1.1 SD in nine patients. Cohen et al. (4) used $12 \mathrm{~Gy}$ f-TBI and reported a mean decrease in height SDS of 0.9 SD in 14 patients. Sanders et al. (8) used 12-15.75 Gy f-TBI and report a mean decrease in height SDS of approximately $1.5 \mathrm{SD}$ in 21 patients receiving TBI before the age of $10 \mathrm{y}$. The only multicenter 
study by Cohen et al. (1) reported similar final height SDS in 39 prepubertal children receiving sf-TBI $(-1.2 \pm 1.1 \mathrm{SD})$ compared with 39 children receiving f-TBI $(-1.0 \pm 1.2 \mathrm{SD})$, although total decrease in height SDS was slightly greater in the patients receiving sf-TBI (1.4 versus $0.9 \mathrm{SD})$. Based on the results of the available final height data, we conclude that sf-TBI and f-TBI may have a similar effect on growth (i.e. mean decrease in height SDS after prepubertal TBI of approximately $1-1.5 \mathrm{SD}$ ).

As in previous studies, decrease in final height was greater in boys (median $1.7 \mathrm{SD}$ versus $1.1 \mathrm{SD}$ in girls). In addition, in boys, most of the decrease in height SDS occurred during puberty, whereas in girls decrease in height SDS was slightly greater before puberty (not significant) and much less during puberty (all puberty-sex interactions, with boys as the reference category, were positive). There are several possible explanations for these differences between boys and girls. First, the time between SCT and onset of puberty was slightly greater in girls (median 2.8 y versus $1.5 \mathrm{y}$ in boys). Second, it is possible that maximum height velocity is limited by radiation-induced structural damage to the growth plates (e.g. to $5-6 \mathrm{~cm} / \mathrm{y})$. As PHV is greater in healthy boys compared with girls, limiting the PHV may have had a greater effect on boys. Third, ovarian failure frequently occurred in girls, whereas Leydig cell failure only occurred in the eight boys who received a testicular booster irradiation. Delayed introduction of sex hormone replacement therapy in girls may have resulted in a prolonged period of prepubertal growth.

Body proportions. Data on body proportions after TBIbased conditioning for SCT are limited. Three studies compared sitting height SDS to leg length SDS (9-11). Two other studies compared sitting height SDS to standing height SDS $(5,12)$. All studies describe a greater impairment of growth of the spine compared with that of the lower limbs, with differences between sitting height SDS and height (or leg length) SDS of 0 SD to -1.5 SD (on average). Most studies, however, included a relatively small number of patients, or patients who had received cranial or craniospinal irradiation, or were treated with GH $(5,9-11)$. In addition, none of the previous studies investigated the differences in body proportions between boys and girls.

In our study, changes in SDS for height, sitting height, leg length, and sitting height/height ratio were investigated in boys and girls separately. In boys, the decreases in SDS for sitting height and leg length were of comparable magnitude. Therefore, sitting height/height ratio SDS did not change. In girls, however, a decrease in sitting height SDS was not accompanied by a decrease in leg length SDS, resulting in a significant decrease in sitting height/height ratio SDS. As estrogen deficiency causes a relative increase in leg length compared with sitting height, we hypothesise that lack of estrogen is responsible for the decrease in sitting height/height ratio SDS in girls. Indeed, the decrease in sitting height/height ratio SDS was more pronounced in girls diagnosed with gonadal failure before the age of $15 \mathrm{y}$. Possible explanations for the differences between our results and those of previous studies are the separate analysis of boys and girls, the exclusion of patients with a history of CNS irradiation or GH treatment, and differences in TBI regimens (most of our patients received a lower total dose of TBI (7-8 Gy sf-TBI) compared with the other studies (9-10 Gy sf-TBI or $>10$ Gy f-TBI).

Modeling growth after SCT. Modeling growth after SCT is complicated by the fact that there are several time variables to consider (i.e. chronological age, time since SCT, and time since onset of puberty), all of which are closely correlated. As we were interested in the effects of TBI and SCT on growth, we chose the SCT as starting point, with time since SCT as our primary time variable. As changes in absolute height in normally growing children are influenced by chronological age, we chose changes in height SDS as our main outcome variable. The effect of puberty on changes in height SDS was separated into the effect of the pubertal growth spurt in the reference population and the effect of the patients' individual pubertal growth spurt (as mentioned in "Methods"). On empirical basis, we have used nonlinear effects to account for these different effects of puberty. The other parameters were added to the model as linear effects. The parameters "time since SCT" and "individual puberty" were added to the model with both fixed and random effects. The final model shows that, in our patient population, there is a constant decrease in height SDS with time after TBI-based conditioning for SCT, with no significant sex difference. It also shows a slight attenuation of this constant decrease with increasing age at $\mathrm{SCT}$, even though the TBI dose was lower in younger children. This suggests that younger children are more vulnerable to the growth-limiting effects of radiation. During puberty, the increase in height SDS as a result of the individual pubertal growth spurt of our patients was slightly greater in girls compared with boys (Fig. 2, $c$ and f), but the difference was not significant. In boys (but not in girls), the individual pubertal growth spurt could not compensate for the loss of height SDS caused by the pubertal growth spurt in the reference population, leading to an accelerated loss of height SDS during puberty. As the logistic component of the negative effect of the pubertal growth spurt in the reference population was greater in boys compared with girls, the greater loss of height SDS during puberty in boys was more likely to be the result of the greater pubertal growth spurt in the reference population than to a lower individual growth spurt in the boys in our study. Indeed, median pubertal PHV after SCT was slightly higher in boys compared with girls after TBI, but the difference between the sexes $(0.5 \mathrm{~cm} / \mathrm{y})$ was less than observed between boys and girls in the normal population $(>1 \mathrm{~cm} / \mathrm{y})$.

This study shows that growth and body proportions after TBI and SCT are affected in a sex-specific way. It identifies several influencing factors, which may help to clarify mechanisms behind growth delay after TBI and SCT. Moreover, this model of growth after TBI provides us with a powerful tool for future evaluations of the effect of growth hormone treatment on growth after TBI.

\section{REFERENCES}

1. Cohen A, Rovelli A, Bakker B, Uderzo C, van Lint, MT, Esperou H, Gaiero A, Leiper AD, Dopfer R, Cahn JY, Merlo F, Kolb HJ, Socie G 1999 Final height of 
patients who underwent bone marrow transplantation for hematological disorders during childhood: a study by the Working Party for Late Effects-EBMT. Blood 93:4109-4115

2. Darzy KH, Shalet SM 2003 Radiation-induced growth hormone deficiency. Horm Res 59 suppl 1:1-11

3. Clement-de Boers A, Oostdijk W, Van-Weel-Sipman MH, Van-den-Broeck J, Wit JM, Vossen JM 1996 Final height and hormonal function after bone marrow transplantation in children. J Pediatr 129:544-550

4. Cohen A, Rovelli A, van Lint MT, Uderzo C, Morchio A, Pezzini C, Masera G, Bacigalupo A, Romano C 1996 Final height of patients who underwent bone marrow transplantation during childhood. Arch Dis Child 74:437-440

5. Holm K, Nysom K, Rasmussen MH, Hertz H, Jacobsen N, Skakkebaek NE, Krabbe S, Muller J 1996 Growth, growth hormone and final height after BMT. Possible recovery of irradiation-induced growth hormone insufficiency. Bone Marrow Transplant 18:163-170

6. Bakker B, Massa GG, Oostdijk W, Weel-Sipman MH, Vossen JM, Wit JM 2000 Pubertal development and growth after total-body irradiation and bone marrow transplantation for haematological malignancies. Eur J Pediatr 159:31-37

7. Frisk P, Arvidson J, Gustafsson J, Lonnerholm G 2004 Pubertal development and final height after autologous bone marrow transplantation for acute lymphoblastic leukemia. Bone Marrow Transplant 33:205-210

8. Sanders JE, Guthrie KA, Hoffmeister PA, Woolfrey AE, Carpenter PA, Appelbaum FR 2005 Final adult height of patients who received hematopoietic cell transplantation in childhood. Blood 105:1348-1354

9. Leiper AD, Stanhope R, Lau T, Grant DB, Blacklock H, Chessells JM, Plowman PN 1987 The effect of total body irradiation and bone marrow transplantation during childhood and adolescence on growth and endocrine function. $\mathrm{Br} \mathrm{J}$ Haematol 67:419-426
10. Papadimitriou A, Urena M, Hamill G, Stanhope R, Leiper AD 1991 Growth hormone treatment of growth failure secondary to total body irradiation and bone marrow transplantation. Arch Dis Child 66:689-692

11. Thomas BC, Stanhope R, Plowman PN, Leiper AD 1993 Growth following single fraction and fractionated total body irradiation for bone marrow transplantation. Eur J Pediatr 152:888-892

12. Brauner R, Adan L, Souberbielle JC, Esperou H, Michon J, Devergie A, Gluckman E, Zucker JM 1997 Contribution of growth hormone deficiency to the growth failure that follows bone marrow transplantation. J Pediatr 130:785-792

13. Tanner JM, Whitehouse RH 1976 Clinical longitudinal standards for height, weight, height velocity, weight velocity, and stages of puberty. Arch Dis Child 51:170-179

14. Fredriks AM, van Buuren S, Burgmeijer RJ, Meulmeester JF, Beuker RJ, Brugman E, Roede MJ, Verloove-Vanhorick SP, Wit JM 2000 Continuing positive secular growth change in The Netherlands 1955-1997. Pediatr Res 47:316-323

15. Fredriks AM, van Buuren, S, van Heel, WJ, Dijkman-Neerincx RH, VerlooveVanhorick SP, Wit JM 2005 Nationwide age references for sitting height, leg length, and sitting height/height ratio, and their diagnostic value for disproportionate growth disorders. Arch Dis Child 90:807-812

16. Ramsay JO, Bock RD, Gasser T 1995 Comparison of height acceleration curves in the Fels, Zurich, and Berkeley growth data. Ann Hum Biol 22:413-426

17. Ramsay JO, Silverman BW 2002 Zooming in on Human Growth. In: Ramsay JO, Silverman BW (eds) Applied Functional Data Analysis: Methods and Case Studies. Springer-Verlag, New York, pp 83-100

18. Beunen G, Malina RM 1988 Growth and physical performance relative to the timing of the adolescent spurt. Exerc Sport Sci Rev 16:503-540

19. Sanders JE 1999 Growth and development after hematopoietic cell transplantation. In: Thomas ED, Blume KG, Forman SJ (eds) Hematopoietic Cell Transplantation. Blackwell Science, Malden, UK, pp 764-775. 